\title{
CONSORT extension for reporting N-of-1 trials (CENT) 2015 Statement
}

\begin{abstract}
Sunita Vohra, ${ }^{1}$ Larissa Shamseer, ${ }^{2}$ Margaret Sampson, ${ }^{3}$ Cecilia Bukutu, ${ }^{4}$ Christopher H Schmid, ${ }^{5}$ Robyn Tate, ${ }^{6}$ Jane Nikles, ${ }^{7}$ Deborah R Zucker, ${ }^{8}$ Richard Kravitz,, ${ }^{9}$ Gordon Guyatt, ${ }^{10}$ Douglas G Altman,11 David Moher ${ }^{2}$ and the CENT group
\end{abstract}

UUniversity of Alberta, Canada

${ }^{2}$ Ottawa Hospital Research Institute, and University of

Ottawa, Canada

'Library Services, Children's Hospital of Eastern Ontario, Canada

${ }^{4}$ Alberta Centre for Child, Family and Community Research,

Canada

${ }^{5}$ Center for Evidence Based Medicine, Brown University, USA

${ }^{6}$ Centre for Rehabilitation Research, Sydney Medical School - Northern, University of Sydney, Australia

7University of Queensland, Australia

8Tufts University School of Medicine, USA

Unniversity of California, Davis, USA

${ }^{10}$ McMaster University, Canada

${ }^{11}$ Centre for Statistics in Medicine, University of Oxford, UK

Correspondence to: S Vohra svohra@ualberta.ca

Cite this as: $B M J$ 2015;350:h1738 doi: 10.1136/bmj.h1738

Accepted: 06 January 2015
N-of-1 trials provide a mechanism for making evidence based treatment decisions for an individual patient. They use key methodological elements of group clinical trials to evaluate treatment effectiveness in a single patient, for situations that cannot always accommodate large scale trials: rare diseases, comorbid conditions, or in patients using concurrent therapies. Improvement in the reporting and clarity of methods and findings in N-of- 1 trials is essential for reader to gauge the validity of trials and to replicate successful findings. A CONSORT extension for $\mathrm{N}$-of- 1 trials (CENT 2015) provides guidance on the reporting of individual and series of $\mathrm{N}$-of-1 trials. CENT provides additional guidance for 14 of the 25 items of the CONSORT 2010 checklist and recommends a diagram for depicting an individual N-of-1 trial and modifies the CONSORT flow diagram to address the flow of a series of $\mathrm{N}$-of-1 trials. The rationale, development process, and CENT 2015 checklist and diagrams are reported in this document.

Although randomised controlled trials are the ideal for establishing treatment efficacy, they have limitations. ${ }^{12}$ In particular, even the most rigorous and clearly reported randomised controlled trial cannot predict if a given intervention will be effective in a specific individual. For this reason, $\mathrm{N}$-of- 1 trials have been placed, by some, on the pinnacle of the evidence hierarchy for making decisions about treatment benefits and harms. ${ }^{34}$ They use key methodological elements of group clinical trials to evaluate treatment effectiveness in a single patient, for situations that cannot always accommodate large scale trials: rare diseases, comorbid conditions, or in patients using concurrent therapies.

$\mathrm{N}$-of- 1 trials have been used to evaluate a range of health conditions-including mental and behavioural disorders and diseases of the nervous, respiratory, circulatory, musculoskeletal, and digestive systems ${ }^{5}-$ and are appropriate for a range of interventions, pharmacological or non-pharmacological, including complementary or alternative therapies. However, $\mathrm{N}$-of- 1 trials are not applicable for all health conditions or interventions. They are ideal for chronic stable conditions and for therapies with a relatively quick onset of action after initiation and quick termination of effect after discontinuation.

In an era that promotes patient centered research, comparative effectiveness, and personalised medicine, $\mathrm{N}$-of-1 trials allow clinicians and patients to evaluate health interventions in a rigorous fashion and to re-evaluate chronic therapies to ensure therapeutic effectiveness is still achieved. $\mathrm{N}$-of- 1 trials are a unique tool to elicit patient preferences and to facilitate shared decision-making, hence evidence-based medicine, in real clinical practice. In addition, $\mathrm{N}$-of-1 trials may also be used to assess causality of potential adverse effects. By reducing ineffective polypharmacy, N-of-1 trials may help promote patient safety.

\section{N-of-1 methodology and reporting}

N-of-1 trials provide a methodologically rigorous evaluation of treatment effectiveness in a single individual at a time, thereby helping to close the gap between evidence and practice. Unlike randomised controlled trials, which often exclude patients with comorbid conditions or concurrent therapies, N-of- 1 trials allow a more flexible approach. Patients may be included as long as their health and concurrent therapies are stable during the period of evaluation; caution is necessary if combining results of N-of-1 trials because of heterogeneity between patients. As in group crossover trials, the potential for confounding by covariates is eliminated in N-of- 1 trials given that each patient serves as his or her own control. The use of multiple crossovers in N-of- 1 trials also increases confidence in the reliability of the results.

A recent systematic review of 100 trial reports indicates that the reporting of N-of-1 trials is largely inadequate. ${ }^{5}$ As an example, $79 \%$ of reports included in the review did not indicate which outcome was considered to be primary. Another $64 \%$ of reports did not comment on the presence or absence of adverse 
events. If N-of- 1 trials are to be useful, these and other essential pieces of information must be included.

Among the first initiatives aiming to improve the completeness of reporting of research studies was the Consolidated Standards of Reporting Trials (CONSORT) guideline, which first emerged in $1996^{6}$ and has been revised twice, most recently in $2010,{ }^{78}$ to keep in line with evolving evidence and methodological standards for trials. Extensions to the main CONSORT document have been developed to address the reporting of variations in trial designs, ${ }^{9-11}$ interventions, ${ }^{12-14}$ and data. ${ }^{15-17}$ The CENT guideline provides a set of items, modified from the CONSORT 2010 guideline to facilitate complete reporting of N-of-1 trials. The development of CENT 2015 is in line with recent, international efforts to improve the reporting of research overall. ${ }^{18}$

For convenience, throughout this document, we refer to treatments and patients, although we recognise that not all interventions evaluated in N-of- 1 trials are technically treatments and that participants are not always patients.

\section{Terminology and scope of CENT}

The single case experimental design, including N-of-1 trials, has a long history in the behavioural sciences. ${ }^{1920}$ In the medical literature, the term "N-of- 1 trial" is used to describe a prospectively planned, multiple crossover trial in a single individual-often challenge-withdrawal-challenge-withdrawal, also described as "ABAB". ${ }^{20}$ CENT restricts itself to this use of the term "N-of-1", rather than all possible single case experimental designs (fig 1), for which a separate reporting guideline is under development. ${ }^{21}$ Consisting of repeated units of treatment comparisons (see box 1), N-of-1 trials may compare an active treatment against placebo, more than one active treatment, or perhaps multiple doses of a single treatment. The comparison depends on the question that is being asked. Definitions of methodological terminology specific to $\mathrm{N}$-of- 1 trials are provided in box 1 .

Since approximately $60 \%$ of N-of- 1 trial publications report on results from more than a single patient, ${ }^{5}$ the CENT reporting guidelines have been designed to facilitate the reporting of both a single N-of- 1 trial or a prospectively planned series of multiple N-of- 1 trials. Readers may also wish to use the checklist to facilitate

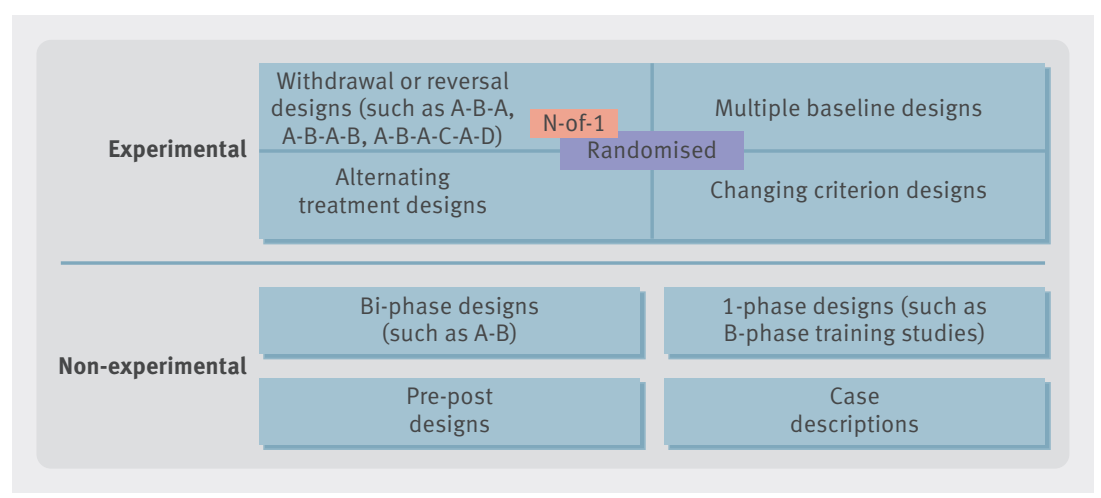

Fig 1 | Common single case designs. CENT is applicable to a subset of the "Withdrawal/ reversal designs" category, which may or may not include the use of randomisation, designated by the red "N-of-1" box (adapted from ${ }^{27}$ ) the critical appraisal of published N-of-1 trial reports for completeness and transparency. This guidance is not intended to be applied by those reporting systematic reviews in which N-of-1 trials are included; future such guidance is planned.

\section{Developing the CENT Statement}

An international steering committee (DGA, Nick Barrowman, CB, GG, DM, JN, MS, RT, SV) comprising members with wide experience in clinical trial methodology and reporting guideline development, together with the project coordinator (LS), led the development of CENT. Two members of the team are practising physicians (SV and JN), and two others are members of the CONSORT and EQUATOR (Enhanced Quality and Transparency of Reporting) Network executive (DGA and DM). The EQUATOR Network is a group dedicated to "improving the reliability and value of medical research literature by promoting transparent and accurate reporting of research studies" (www.equator-network.org). The CENT Statement was developed in

Box 1: Methodological terminology typical in N-of-1 trial reports

- $\mathrm{N}$-of-1 trial-An experimental clinical study design to determine the effect of an intervention in a single study participant. CENT is intended to be used to report repeated challenge-withdrawal (that is, "ABAB") trials, commonly used in medicine, in which multiple crossovers between treatment(s) and control (placebo, standard care, alternate treatment) are continued for a pre-specified amount of time or until treatment effectiveness is determined. More than two treatment alternatives may be compared to each other or control (that is, "ABCABC")

- Period-The time during which a single treatment (A or B) is administered. Period length is typically determined a priori and may vary within a trial. The order of periods within a pair or treatment block may be randomised.

- Block or pair-A repeated unit of a set number of period in N-of-1 trials is referred to as a block, in which the sequence of periods may or may not be randomised (for example, three repeating blocks of four periods may look like "AABB BBAA ABAB"). By convention, when the repeated unit contains only two periods (for example, three repeating pairs may look like " $A B B A$ BA"), it is conventionally referred to as a pair.

- Sequence-Multiple pairs or blocks comprise an entire sequence. The sequence is the consecutive set of periods, which may or may not indicate size of the repeated unit.

- Washout period-A period in which no intervention is administered. A washout may be administered between different treatment periods or may act as a period in itself, as in a "reversal" design (to "wash out" the effects of a treatment before it is re-administered).

- Run-in period-A pre-specified duration of time before a trial begins, during which trial treatments may be initiated (for example, to get to a stable therapeutic dose), to determine potential patient compliance with study regimens, or to allow for washout of a medication(s) a patient may have been taking before the trial. 
accordance with the process developed by members of EQUATOR executive group. ${ }^{22}$

\section{Pre-meeting activities}

In order to assess whether a reporting guideline for $\mathrm{N}$-of-1 trials was indeed warranted, members of the CENT group (CB, MS, LS, SV) undertook a systematic review to assess the design and reporting of N-of- 1 trials in the medical literature. ${ }^{5}$ This work confirmed the heterogeneity in N-of- 1 trials, and large inadequacies in their reporting, as described above.

Between February 2009 and April 2009, a modified Delphi process was carried out to seek consensus on a set of potential reporting items $(\mathrm{n}=55)$ for $\mathrm{N}$-of- 1 trials. This initial list was developed based on the characteristics of published trials guided by our systematic review and reporting elements from the CONSORT 2010 checklist. ${ }^{7}$ A two-round survey was sent out to 56 international experts of N-of-1 trialists, biostatisticians, clinical epidemiologists, reporting guideline developers, biomedical journal editors, and health research funders, of whom $75 \%$ and $62 \%$ responded in rounds 1 and 2, respectively. Participants were asked to rank the importance of each potential checklist item on a 1 to 10 scale. After both rounds of surveying, items with a mean ranking of $\leq 5$ were excluded from further consideration on the CENT checklist. Items ranking $\geq 6$ or for which there was a large discrepancy between participants were carried forward for further discussion at the CENT consensus meeting.

\section{CENT consensus meeting}

In May 2009, a two day, in person meeting was convened in Banff, Canada, during which 23 experts in N-of-1 methodology, guideline development, N-of-1 study funders, and journal editors came together to further discuss and refine proposed checklist items. Discussion at the meeting yielded agreement on a set of essential concepts to be included in the guidelines; nuances of wording were not discussed at the meeting.

\section{Post-meeting activities}

After the CENT meeting, wording and organisation of concepts into checklist items was carried out and refined within the steering group and approved by meeting participants. A second round of circulation solicited feedback from those invited to the meeting but unable to attend. A flow diagram subcommittee was formed (NBG, JN, DRZ) to lead development of the CENT flow diagrams.

\section{CENT 2015 checklist}

The final CENT 2015 checklist is an extension of 14 items of the 25 CONSORT 2010 (table 1) and contains a total of 44 sub-items, some of which are modifications of a standard CONSORT 2010 item and some of which are in addition. For item $1 \mathrm{~b}$, pertaining to the abstract, recommendations specific for N-of-1 trials are proposed in table 2 . Since $\mathrm{N}$-of- 1 trials may be conducted for an individual or a series of individuals, where pertinent, the checklist indicates specific items where different or additional information is required for series of N-of- 1 trials. The recommendations within CENT may require more words or space than N-of-1 trialists are accustomed to. Providing detailed descriptions for some trials will facilitate transparency and future reproducibility, in line with emerging journal policies aimed at facilitating reproducibility. ${ }^{23}$

We recognise that improved reporting must be balanced against patient confidentiality in situations when the condition is rare. Authors must be mindful of this, and if there is any doubt as to whether complete reporting could be identifying, they should seek consultation with their institutional ethics board. This issue is of heightened importance in N-of-1 trials of rare conditions or when the potential societal stigma is high. Caution should be taken when reporting a combination of identifying information pertaining to CENT items $4 \mathrm{a}$, 4b, $14 \mathrm{a}$, and 15.

\section{CENT diagrams}

Diagrams have been developed to help authors visually depict both an individual participant's progress through an N-of-1 trial (fig 2, CENT trial pictorial) and the flow of multiple participants through a series of trials (fig 3, CENT flow diagram). Authors are encouraged in include the relevant diagram(s) in their N-of-1 reports. Specific guidance on the information that should be included in each diagram is provided in the CENT Explanation and Elaboration document. ${ }^{24}$

\section{CENT 2015 Explanation and Elaboration (E\&E) document}

Once the CENT checklist was drafted, the steering committee and members from the larger CENT group prepared a rationale and examples of good reporting for the final set of checklist items. These can be found in the CENT 2015 Explanation and Elaboration (E\&E) document. $^{24}$ The principles addressed in this document were largely derived from existing literature, the CONSORT 2010 E\&E document ${ }^{8}$ where applicable, and from discussions at the CENT meeting, which was recorded and summarised.

We strongly recommend that authors preparing N-of-1 trial reports and those charged with reviewing them use the CENT 2015 checklist to guide or assess their reporting. Authors are urged to read and use the CENT 2015 E\&E document together with the checklist, as it provides examples of good reporting and evidence and consensus based rationale and guidance on how to report each item. ${ }^{24}$

\section{Post-publication activities}

Post-publication activities are planned around CENT 2015, including collecting feedback from the broader scientific community on the CENT initiative and specific checklist items. We also plan to seek endorsement from medical journals known to publish N-of-1 trials (see below). The CENT 2015 Checklist, Statement, and E\&E documents are available through the CONSORT website 


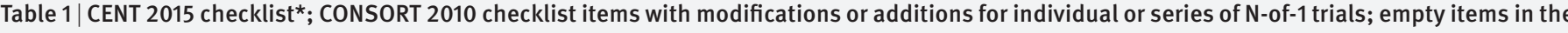
CENT 2015 column indicate no modification from the CONSORT 2010 item

CONSORT 2010

Section/Topic

No Item

Title and abstract

\section{CENT 2015}

No Item

1a Identify as an "N-of-1 trial" in the title

For series: Identify as "a series of $\mathrm{N}$-of-1 trials" in the title

1b For specific guidance, see CENT guidance for abstracts (table 2)

1b Structured summary of trial design, methods, results, and conclusions (for specific guidance see CONSORT for abstracts)

\section{Introduction}

Background and objectives

2a Scientific background and explanation of rationale

2 a.1

$2 \mathrm{~b} \quad$ Specific objectives or hypotheses

\begin{tabular}{lll}
\hline Methods & 3a & $\begin{array}{l}\text { Description of trial design (such as parallel, factorial) } \\
\text { including allocation ratio }\end{array}$
\end{tabular}

3b Important changes to methods after trial start (such as eligibility criteria), with reasons

Participant(s)

4a Eligibility criteria for participants

4b Settings and locations where the data were collected

$3 a$

Rationale for using N-of-1 approach

b

Rationale for using $\mathrm{N}$-of-1 approach

3a

Describe trial design, planned number of periods, and duration of each period (including run-in and wash out, if applicable) In addition for series: Whether and how the design was individualized to each participant, and explain the series design

$3 b$

$3 b$

4at
Diagnosis or disorder, diagnostic criteria, comorbid conditions,
and concurrent therapies.

For series: Same as CONSORT item $4 \mathrm{a}$

4c Whether the trial(s) represents a Research Methods \& Reporting study and if so, whether institutional ethics approval was obtained

\begin{tabular}{|c|c|c|c|c|}
\hline Interventions & 5 & $\begin{array}{l}\text { The interventions for each group with sufficient details to allow } \\
\text { replication, including how and when they were actually } \\
\text { administered }\end{array}$ & 5 & $\begin{array}{l}\text { The interventions for each period with sufficient details to allow } \\
\text { replication, including how and when they were actually } \\
\text { administered }\end{array}$ \\
\hline \multirow[t]{3}{*}{ Outcomes } & $6 a$ & $\begin{array}{l}\text { Completely defined pre-specified primary and secondary } \\
\text { outcome measures, including how and when they were } \\
\text { assessed }\end{array}$ & $6 a .1$ & \\
\hline & & & $6 a .2$ & $\begin{array}{l}\text { Description and measurement properties (validity and reliability) } \\
\text { of outcome assessment tools }\end{array}$ \\
\hline & $6 b$ & $\begin{array}{l}\text { Any changes to trial outcomes after the trial commenced, with } \\
\text { reasons }\end{array}$ & $6 b$ & \\
\hline
\end{tabular}

Sample size

\begin{tabular}{lll}
$7 \mathrm{a}$ & How sample size was determined & $7 \mathrm{a}$ \\
\hline $7 \mathrm{~b}$ & $\begin{array}{l}\text { When applicable, explanation of any interim analyses and } \\
\text { stopping guidelines }\end{array}$ & $7 \mathrm{~b}$
\end{tabular}

\begin{tabular}{|c|c|c|c|c|}
\hline \multicolumn{5}{|l|}{ Randomisation: } \\
\hline \multirow[t]{3}{*}{ Sequence generation } & $8 a$ & Method used to generate the random allocation sequence & $8 a$ & $\begin{array}{l}\text { Whether the order of treatment periods was randomised, with } \\
\text { rationale, and method used to generate allocation sequence }\end{array}$ \\
\hline & $8 b$ & $\begin{array}{l}\text { Type of randomisation; details of any restriction (such as } \\
\text { blocking and block size) }\end{array}$ & $8 b$ & $\begin{array}{l}\text { When applicable, type of randomisation; details of any } \\
\text { restrictions (such as pairs, blocking) }\end{array}$ \\
\hline & & & $8 c$ & Full, intended sequence of periods \\
\hline $\begin{array}{l}\text { Allocation concealment } \\
\text { mechanism }\end{array}$ & 9 & $\begin{array}{l}\text { Mechanism used to implement the random allocation } \\
\text { sequence (such as sequentially numbered containers), } \\
\text { describing any steps taken to conceal the sequence until } \\
\text { interventions were assigned }\end{array}$ & 9 & \\
\hline Implementation & 10 & $\begin{array}{l}\text { Who generated the random allocation sequence, who enrolled } \\
\text { participants, and who assigned participants to interventions }\end{array}$ & 10 & \\
\hline \multirow[t]{2}{*}{ Blinding } & $11 \mathrm{a}$ & $\begin{array}{l}\text { If done, who was blinded after assignment to interventions (for } \\
\text { example, participants, care providers, those assessing } \\
\text { outcomes) and how }\end{array}$ & $11 \mathrm{a}$ & \\
\hline & $11 b$ & If relevant, description of the similarity of interventions & $11 \mathrm{~b}$ & \\
\hline \multirow[t]{3}{*}{ Statistical methods } & $12 a$ & $\begin{array}{l}\text { Statistical methods used to compare groups for primary and } \\
\text { secondary outcomes }\end{array}$ & $12 \mathrm{a}$ & $\begin{array}{l}\text { Methods used to summarize data and compare interventions for } \\
\text { primary and secondary outcomes }\end{array}$ \\
\hline & $12 b$ & $\begin{array}{l}\text { Methods for additional analyses, such as subgroup analyses } \\
\text { and adjusted analyses }\end{array}$ & $12 b$ & $\begin{array}{l}\text { For series: If done, methods of quantitative synthesis of individual } \\
\text { trial data, including subgroup analyses, adjusted analyses, and } \\
\text { how heterogeneity between participants was assessed (for } \\
\text { specific guidance on reporting syntheses of multiple trials, please } \\
\text { consult the PRISMA Statement) }\end{array}$ \\
\hline & & & $12 c$ & $\begin{array}{l}\text { Statistical methods used to account for carryover effect, period } \\
\text { effects, and intra-subject correlation }\end{array}$ \\
\hline
\end{tabular}

Whether the order of treatment periods was randomised, with rationale, and method used to generate allocation sequence

b When applicable, type of randomisation; details of any restrictions (such as pairs, blocking)

8c Full, intended sequence of periods 


\begin{tabular}{|c|c|c|c|c|}
\hline \multirow[b]{2}{*}{ Section/Topic } & \multicolumn{2}{|c|}{ CONSORT 2010} & \multicolumn{2}{|c|}{ CENT 2015} \\
\hline & No & Item & No & Item \\
\hline \multicolumn{5}{|l|}{ Results } \\
\hline \multirow{3}{*}{$\begin{array}{l}\text { Participant flow (a } \\
\text { diagram is strongly } \\
\text { recommended) }\end{array}$} & \multirow[t]{2}{*}{$13 \mathrm{a}$} & \multirow{2}{*}{$\begin{array}{l}\text { For each group, the numbers of participants who were } \\
\text { randomly assigned, received intended treatment, and were } \\
\text { analysed for the primary outcome }\end{array}$} & 13a.1 & $\begin{array}{l}\text { Number and sequence of periods completed, and any changes } \\
\text { from original plan with reasons }\end{array}$ \\
\hline & & & 13a.2 & $\begin{array}{l}\text { For series: The number of participants who were enrolled, } \\
\text { assigned to interventions, and analysed for the primary outcome }\end{array}$ \\
\hline & $13 b$ & $\begin{array}{l}\text { For each group, losses and exclusions after randomisation, } \\
\text { together with reasons }\end{array}$ & $13 c$ & $\begin{array}{l}\text { For series: Losses or exclusions of participants after treatment } \\
\text { assignment, with reasons, and period in which this occurred, if } \\
\text { applicable }\end{array}$ \\
\hline \multirow[t]{2}{*}{ Recruitment } & $14 \mathrm{a}$ & Dates defining the periods of recruitment and follow-up & \multicolumn{2}{|l|}{ 14at } \\
\hline & $14 b$ & Why the trial ended or was stopped & $14 b$ & $\begin{array}{l}\text { Whether any periods were stopped early and/or whether trial was } \\
\text { stopped early, with reason(s). }\end{array}$ \\
\hline Baseline data & 15 & $\begin{array}{l}\text { A table showing baseline demographic and clinical } \\
\text { characteristics for each group }\end{array}$ & $15 \dagger$ & \\
\hline Numbers analysed & 16 & $\begin{array}{l}\text { For each group, number of participants (denominator) } \\
\text { included in each analysis and whether the analysis was by } \\
\text { original assigned groups }\end{array}$ & 16 & $\begin{array}{l}\text { For each intervention, number of periods analysed. } \\
\text { In addition for series: If quantitative synthesis was performed, } \\
\text { number of trials for which data were synthesized }\end{array}$ \\
\hline \multirow[t]{3}{*}{$\begin{array}{l}\text { Outcomes and } \\
\text { estimation }\end{array}$} & \multirow[t]{2}{*}{$17 a$} & \multirow{2}{*}{$\begin{array}{l}\text { For each primary and secondary outcome, results for each } \\
\text { group, and the estimated effect size and its precision (such as } \\
95 \% \text { confidence interval) }\end{array}$} & 17a.1 & $\begin{array}{l}\text { For each primary and secondary outcome, results for each period; } \\
\text { an accompanying figure displaying the trial data is recommended. }\end{array}$ \\
\hline & & & 17a.2 & $\begin{array}{l}\text { For each primary and secondary outcome, the estimated effect } \\
\text { size and its precision (such as } 95 \% \text { confidence interval) } \\
\text { In addition for series: If quantitative synthesis was performed, } \\
\text { group estimates of effect and precision for each primary and } \\
\text { secondary outcome }\end{array}$ \\
\hline & $17 b$ & $\begin{array}{l}\text { For binary outcomes, presentation of both absolute and } \\
\text { relative effect sizes is recommended }\end{array}$ & $17 \mathrm{~b}$ & \\
\hline Ancillary analyses & 18 & $\begin{array}{l}\text { Results of any other analyses performed, including subgroup } \\
\text { analyses and adjusted analyses, distinguishing pre-specified } \\
\text { from exploratory }\end{array}$ & 18 & $\begin{array}{l}\text { Results of any other analyses performed, including assessment } \\
\text { of carryover effects, period effects, intra-subject correlation } \\
\text { In addition for series: If done, results of subgroup or sensitivity } \\
\text { analyses }\end{array}$ \\
\hline Harms & 19 & $\begin{array}{l}\text { All important harms or unintended effects in each group (for } \\
\text { specific guidance see CONSORT for harms) }\end{array}$ & 19 & $\begin{array}{l}\text { All harms or unintended effects for each intervention. (for specific } \\
\text { guidance see CONSORT for harms) }\end{array}$ \\
\hline \multicolumn{5}{|l|}{ Discussion } \\
\hline Limitations & 20 & $\begin{array}{l}\text { Trial limitations, addressing sources of potential bias, } \\
\text { imprecision, and, if relevant, multiplicity of analyses }\end{array}$ & 20 & \\
\hline Generalisability & 21 & $\begin{array}{l}\text { Generalisability (external validity, applicability) of the trial } \\
\text { findings }\end{array}$ & 21 & \\
\hline Interpretation & 22 & $\begin{array}{l}\text { Interpretation consistent with results, balancing benefits and } \\
\text { harms, and considering other relevant evidence }\end{array}$ & 22 & \\
\hline \multicolumn{5}{|l|}{ Other information } \\
\hline Registration & 23 & Registration number and name of trial registry & 23 & \\
\hline Protocol & 24 & Where the full trial protocol can be accessed, if available & 24 & \\
\hline Funding & 25 & $\begin{array}{l}\text { Sources of funding and other support (such as supply of } \\
\text { drugs), role of funders }\end{array}$ & 25 & \\
\hline
\end{tabular}

* It is strongly recommended that this checklist be read in conjunction with the CENT 2015 Explanation and Elaboration ${ }^{24}$ for important clarification on the items. The copyright for CENT (including checklist) is held by the CENT Group and is distributed under a Creative Commons Attribution (CC-BY 4.0) license.

tCaution should be taken when reporting potentially identifying information pertaining to CENT items 4a, 4b, 14a, and 15 .

(www.consort-statement.org) and accessible through the Enhancing the Quality and Transparency of Health Research (EQUATOR) Network library of reporting guidelines (http://www.equator-network.org/ resource-centre/library-of-health-research-reporting/).

\section{Endorsement}

The CONSORT Statement is likely the best known reporting guideline. It has been well received by several scientific organisations (including the International Committee of Medical Journal Editors, Committee on Publication Ethics, and World Association of Medical Editors) and many biomedical journals, over 600 of which currently endorse CONSORT. Endorsement is typically demonstrated by way of an editorial statement in a journal's "Instructions to authors" section, supporting the use of one or more reporting guidelines. Following this model, we plan to seek endorsement from journals that publish N-of- 1 trials and work with these journals to ensure author adherence to reporting CENT 2015 items. To help journals ensure optimal use of the CENT 2015 by authors, we propose the following wording:

“[This journal] requires a completed CENT 2015 checklist as a condition of submission of reports of individual N-of- 1 trials or a series of N-of- 1 trials. We recommend that, while completing this form, you consider amending your manuscript to ensure your article, at a minimum, addresses each item listed on the CENT 2015 checklist. Taking the time to ensure your manuscript meets these basic reporting requirements will greatly improve your manuscript, potentially enhancing its chances for eventual publication.” 


\begin{tabular}{|c|c|c|}
\hline Item & CONSORT for abstracts & Extension for $\mathrm{N}$-of-1 designs \\
\hline Title & Identification of the study as randomised & Identification of the study as an N-of-1 trial or series of $\mathrm{N}$-of- 1 trials in the title \\
\hline Authors* & Contact details for the corresponding author & \\
\hline Trial design & Description of trial design (such as parallel, cluster, non-inferiority) & Description of trial design, number of periods, and period duration \\
\hline \multicolumn{3}{|c|}{ 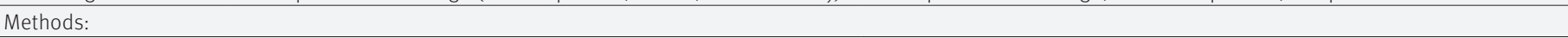 } \\
\hline Participant(s) & $\begin{array}{l}\text { Eligibility criteria for participants and the settings where the data } \\
\text { were collected }\end{array}$ & $\begin{array}{l}\text { For individual trial, clinical condition under study } \\
\text { For series, eligibility criteria for participants }\end{array}$ \\
\hline Interventions & Interventions intended for each group & Interventions intended for each period \\
\hline Objective & Specific objective or hypothesis & \\
\hline Outcome & Clearly defined primary outcome for this report & \\
\hline Randomisation & How participants were allocated to interventions & \\
\hline Blinding (masking) & $\begin{array}{l}\text { Whether participant(s), care givers, and those assessing the } \\
\text { outcomes were blinded to group assignment }\end{array}$ & \\
\hline \multicolumn{3}{|l|}{ Results: } \\
\hline Numbers randomised & Number of participants randomised to each group & $\begin{array}{l}\text { For individual N-of-1 trial, the number and sequence of periods completed } \\
\text { For series, number of individual trials carried out }\end{array}$ \\
\hline Recruitment & Trial status & Not applicable \\
\hline Numbers analysed & Number of participants analysed in each group & $\begin{array}{l}\text { For individual N-of- } 1 \text { report, number of periods analysed for each intervention } \\
\text { For series, the number of participants analysed }\end{array}$ \\
\hline Outcome & $\begin{array}{l}\text { For the primary outcome, a result for each group and the estimated } \\
\text { effect size and its precision }\end{array}$ & \\
\hline Harms & Important adverse events or side-effects. & \\
\hline Conclusions & General interpretation of the results & \\
\hline Trial registration & Registration number and name of trial register, if applicable & \\
\hline Funding & Source of funding & \\
\hline
\end{tabular}

\section{Registering N-of-1 trials}

$\mathrm{N}$-of-1 trials can be prospectively registered in existing trial registries (albeit with some modifications). Eventually, we plan to work with clinicaltrials.gov and all of the primary registries within the WHO Registry Network (www.who.int/ictrp/network/primary/en/index.html), in accordance with the International Clinical Trials Registry Platform, to enable a broader audience to more easily register their $\mathrm{N}$-of- 1 trials. This will help to increase the transparency of N-of- 1 trial reporting and facilitate appraisal of trial reports for potential biases (selective reporting). Although it is possible to register $\mathrm{N}$-of-1 trials in existing registries few published N-of-1 trials have been registered or indicate being registered in their published report.

\section{Evaluation}

As with other reporting guidelines, the impact of CENT can be evaluated by comparing the completeness of

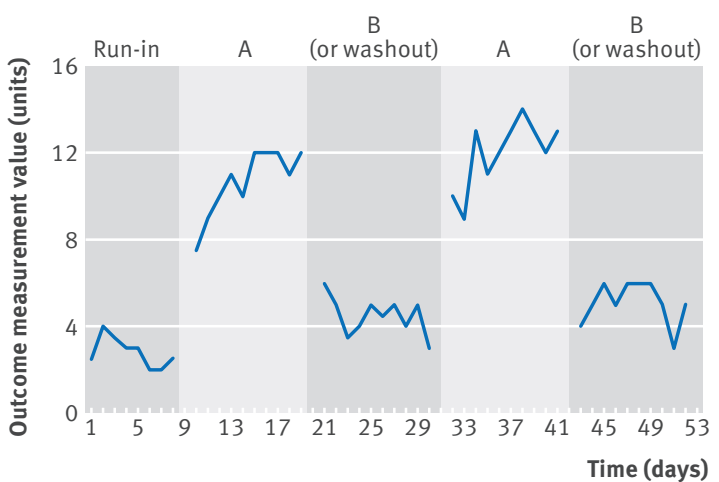

Fig 2 | N-of-1 trial pictorial; suggested visual representation of data from an individual $\mathrm{N}$-of-1 trial reporting of checklist items between $\mathrm{N}$-of- 1 trial reports published in journals endorsing and not endorsing the CENT checklist as well as before versus after endorsement in endorsing journals. This approach reflects methods previously used to evaluate CONSORT and other reporting guidelines..$^{25} 26$

\section{Conclusions}

N-of-1 trials offer a pragmatic approach to evidence based clinical practice. Rather than forcing patients into an all or none fit with a rigid parallel group-based trial protocol, $\mathrm{N}$-of- 1 trials can be flexible and adapt around the individ-

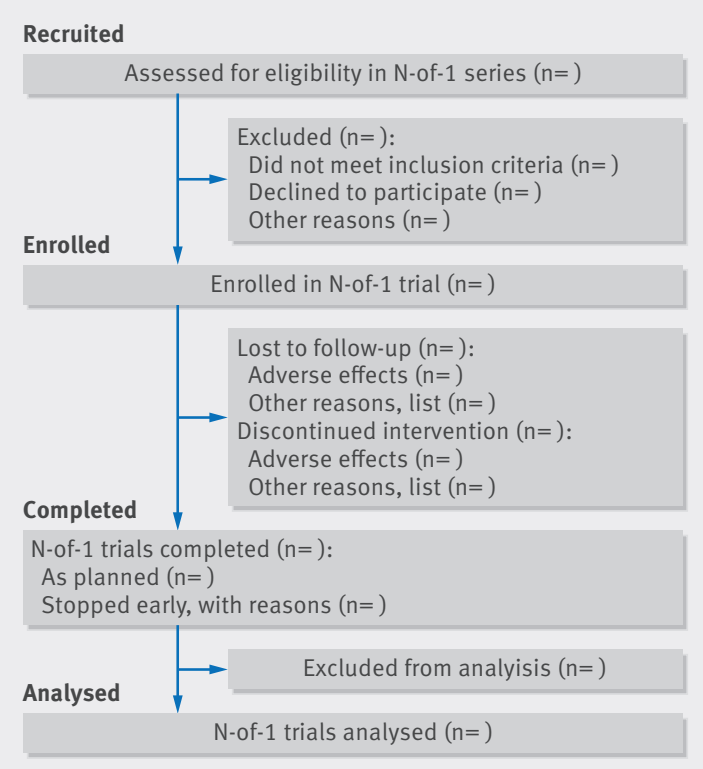

Fig 3 | CENT flow diagram; suggested representation of the flow of participants in a series of $\mathrm{N}$-of- 1 trials 
ual to help assess treatment effectiveness under real world circumstances. They facilitate shared decision making between patients and practitioners, thereby bringing evidence based medicine into real clinical practice. While $\mathrm{N}$-of- 1 trials can be flexible enough for clinical use, they can also be standardised for the purpose of research, which may allow outcomes to be combined for population estimates. By improving the clarity of $\mathrm{N}$-of- 1 trial reporting, the CENT 2015 guidelines offer an opportunity to improve the interpretation and usefulness of N-of-1 trials.

The CENT 2015 guideline is primarily intended to be used by authors and reviewers who report and assess $\mathrm{N}$-of-1 trials, respectively. It may also be helpful earlier in the research process as researchers design protocols for N-of-1 trials.

We thank Kris Cramer for her early work on developing the scope and helping to acquire funding support for this project.

Members of the CENT Group (listed alphabetically):

Douglas G Altman, professor, Centre for Statistics in Medicine, University of Oxford, UK; Cecilia Bukutu, associate director, Child and Youth Data Laboratory, Alberta Centre for Child, Family and Community Research, Canada; Jocalyn Clark, executive editor, icddr,b, Bangladesh; Elise Cogo, research consultant, Canada; Nicole B Gabler, biostatistician, Center for Clinical Epidemiology and Biostatistics Perelman School of Medicine, University of Pennsylvania, USA; Gordon Guyatt, professor, Department of Clinical Epidemiology \& Biostatistics, McMaster University, Canada; Richard Kravitz, professor and co-vice chair of research, Department of Internal Medicine, University of California, Davis, USA; Janine Janosky, vice provost for research, Central Michigan University, USA; Bradley C Johnston, assistant professor, Department of Anesthesia and Pain Medicine, The Hospital for Sick Children, University of Toronto, Canada, and Institute of Health Policy, Management and Evaluation, Dalla Lana School of Public Health, University of Toronto, Canada; Bob Li, scientific advisor (retired), Office of Science, Therapeutic Products Directorate, Health Canada, Canada; Jeff Mahon, professor, Medicine and Epidemiology and Biostatistics, University of Western Ontario, Canada; Robin Marles, senior scientific advisor, Bureau of Nutritional Sciences, Food Directorate, Health Canada, Canada; David Moher, senior scientist, Clinical Epidemiology Program, Ottawa Hospital Research Institute; University of Ottawa, Canada; Jane Nikles, NHMRC postdoctoral research fellow, University of Queensland, Australia; Margaret Sampson, manager, Library Services, Children's Hospital of Eastern Ontario, Canada; Christopher H Schmid, professor of biostatistics, Department of Biostatistics and Center for Evidence Based Medicine, Brown University, USA; William R Shadish, professor, University of California, Merced, USA; Larissa Shamseer, senior research associate, Clinical Epidemiology Program, Ottawa Hospital Research Institute; University of Ottawa, Canada; Robyn Tate, professorial research fellow, Centre for Rehabilitation Research, Sydney Medical School - Northern, University of Sydney, Australia; Sunita Vohra, centennial professor, Department of Pediatrics, Faculty of Medicine and Dentistry, University of Alberta, Canada; Deborah Zucker, adjunct assistant professor, Tufts University School of Medicine, USA.

Contributors: SV, LS, MS, DGA, and DM conceived of this paper. SV and $\mathrm{LS}$ drafted the article and all authors critically revised it for important intellectual content. All authors approved the final version of this article. SV is the guarantor of this work.

Competing interests: All authors have completed the ICMJE uniform disclosure form and declare: the development of CENT was funded by Alberta Advanced Education and Technology, Alberta Heritage Foundation for Medical Research (now Alberta Innovates - Health Solutions (AHIS)), Boiron, CV Technologies (now Afexa Life Sciences), Hecht Foundation, HEEL, Pfizer USA, Schwabe Pharma, and in part, through operational funding awarded by the Canadian Institutes for Health Research (Reference No 86766); no financial relationships with any organisations that might have an interest in the submitted work in the previous three years; representatives from industry partners were present and participated at the CENT consensus meeting and were offered the opportunity to provide input on this manuscript, which none did. SV receives salary support from AHIS as a health scholar. This is an Open Access article distributed in accordance with the Creative Commons Attribution Non Commercial (CC BY-NC 4.0) license, which permits others to distribute, remix, adapt, build upon this work non-commercially, and license their derivative works on different terms, provided the original work is properly cited and the use is noncommercial.
Gitterman DP, Hay WW Jr. That sinking feeling, again? The state of National Institutes of Health pediatric research funding, fiscal year 1992-2010. Pediatr Res 2008:64:462.

2 Fortin M, Dionne J, Pinho G, Gignac J, Almirall J, Lapointe L. Randomized controlled trials: do they have external validity for patients with multiple comorbidities? Ann Fam Med 2006:4:104-8. Guyatt G, Jaeschke R, McGinn T. PART 2B1: therapy and validity. $\mathrm{N}$-of-1 randomized controlled trials. In: Guyatt G, Rennie D, Meade MO, Cook DJ, eds. Users' quides to the medical literature. American Medical Association, 2002:275-90.

4 OCEBM Levels of Evidence Working Group. The Oxford levels of evidence 2. Oxford Centre for Evidence-Based Medicine, 2011. www.cebm.net/index.aspx? $0=5653$.

5 Punja S, Bukutu C, Shamseer L, Sampson M, Hartling L, Urichuk L, et al. Systematic review of the methods, statistical analysis and meta-analysis of N-of-1 trials. University of Alberta, 2014.

6 Begg C, Cho M, Eastwood S, Horton R, Moher D, Olkin I, et al. Improving the quality of reporting of randomized clinical trials: the CONSORT statement. JAMA 1996;276:637-9.

7 Schulz KF, Altman DG, Moher D, for the CONSORT Group. CONSORT 2010 statement: updated guidelines for reporting parallel group randomised trials. PLoS Med 2010;7:e1000251.

8 Moher D, Hopewell S, Schulz KF, Montori V, Gøtzsche PC, Devereaux PJ, et al. CONSORT 2010 explanation and elaboration: updated guidelines for reporting parallel group randomised trials. J Clin Epidemiol 2010;63:e1-37.

9 Zwarenstein M, Treweek S, Gagnier JJ, Altman DG, Tunis S, Haynes B, et al. Improving the reporting of pragmatic trials: an extension of the CONSORT statement. BMJ 2008;337:a2390

10 Campbell MK, Piaggio G, Elbourne DR, Altman DG, CONSORT Group. Consort 2010 statement: extension to cluster randomised trials. BMJ 2012;345:e5661.

11 Piaggio G, Elbourne DR, Pocock SJ, Evans SJ, Altman DG. Reporting of noninferiority and equivalence randomized trials. JAMA 2012;308:2594-604.

12 Gagnier IJ, Boon H, Rochon P, Moher D, Barnes J, Bombardier C, et al. Recommendations for reporting randomized controlled trials of herbal interventions: explanation and elaboration. J Clin Epidemiol 2006:59:1134-49.

13 Boutron I, Moher D, Altman DG, Schulz KF, Ravaud P. Extending the CONSORT statement to randomized trials of nonpharmacologic treatment: explanation and elaboration. Ann Intern Med 2008;148:295.

14 MacPherson H, Altman DG, Hammerschlag R, Li Y, Wu T, White A, et al. Revised standards for reporting interventions in clinical trials of acupuncture (STRICTA): extending the CONSORT statement. Acupuncture Med 2010;28:83.

15 Ioannidis JPA, Evans SJW, Gotzsche PC, O'Neill RT, Altman DG, Schulz $\mathrm{K}$, et al. Better reporting of harms in randomized trials: an extension of the CONSORT statement. Ann Intern Med 2004;141:781-8.

16 Hopewell S, Clarke M, Moher D, Wager E, Middleton P, Altman DG, et al. CONSORT for reporting randomised trials in journal and conference abstracts. Lancet 2008;371:281-3

17 Calvert M, Blazeby J, Altman DG, Revicki DA, Moher D, Brundage MD, et al. Reporting of patient-reported outcomes in randomized trials: the CONSORT PRO extension. JAMA 2013;309:814-22

18 Glasziou P, Altman DG, Bossuyt P, Boutron I, Clarke M, Julious S, et al. Reducing waste from incomplete or unusable reports of biomedical research. Lancet 2014;383:267-76

19 Edgington ES. Statistical inference from $\mathrm{N}=1$ experients. J Psycho 1967;65:195-9.

20 Tate RL, McDonald S, Perdices M, Togher L, Schultz R, Savage S. Rating the methodological quality of single-subject designs and n-of-1 trials: introducing the single-case experimental design (SCED) scale. Neuropsychol Rehabil 2008:18:385-401.

21 Tate R, Togher L, Perdices M, McDonald S, Rosenkoetter U, on behalf of the SCRIBE Steering Committee. Developing reporting guidelines for single-case experimental designs: the SCRIBE project. Brain Impair 2012;13:135.

22 Moher D, Schulz KF, Simera I, Altman DG. Guidance for developers of health research reporting guidelines. PLoS Med 2010;7:e1000217.

23 Enhancing reproducibility. Nature Meth 2013;10:367.

24 Shamseer L, Sampson M, Bukutu C, Schmid CH, Nikles J, Tate R, et al. CONSORT extension for reporting N-of-1 trials (CENT) 2015: Explanation and elaboration. BMJ 2015;350:h1793.

25 Turner L, Shamseer L, Altman DG, Weeks L, Peters J, Kober T, et al. Consolidated standards of reporting trials (CONSORT) and the completeness of reporting of randomized controlled trials published in medical journals: an updated review. Cochrane Database Syst Rev 2012;11:MR000030.

26 Stevens A, Shamseer L, Weinstein E, Yazdi F, Turner L, Thielman J, et al. Relation of completeness of reporting of health research to journals' endorsement of reporting guidelines: systematic review. BMJ 2014;348:g3804

27 Tate RL, Perdices M, Rosenkoetter U, Wakim D, Godbee K, Togher L, et al. Revision of a method quality rating scale for single-case experimental designs and n-of- 1 trials: the 15 -item risk of bias in n-of-1 trials (RoBiNT) scale. Neuropsychol Rehabil 2013;23:619-38. 